\title{
Invited Article: Design techniques and noise properties of ultrastable cryogenically cooled sapphire-dielectric resonator oscillators
}

\author{
C. R. Locke, E. N. Ivanov, J. G. Hartnett, P. L. Stanwix, and M. E. Tobar \\ School of Physics, The University of Western Australia, 35 Stirling Hwy, Crawley, \\ 6009 Western Australia, Australia
}

(Received 9 November 2007; accepted 23 March 2008; published online 12 May 2008)

\begin{abstract}
We review the techniques used in the design and construction of cryogenic sapphire oscillators at the University of Western Australia over the 18 year history of the project. We describe the project from its beginnings when sapphire oscillators were first developed as low-noise transducers for gravitational wave detection. Specifically, we describe the techniques that were applied to the construction of an interrogation oscillator for the PHARAO Cs atomic clock in CNES, in Toulouse France, and to the 2006 construction of four high performance oscillators for use at NMIJ and NICT, in Japan, as well as a permanent secondary frequency standard for the laboratory at UWA. Fractional-frequency fluctuations below $6 \times 10^{-16}$ at integration times between 10 and $200 \mathrm{~s}$ have been repeatedly achieved. (C) 2008 American Institute of Physics. [DOI: 10.1063/1.2919944]
\end{abstract}

\section{INTRODUCTION}

Inspired by the early work of Braginsky et al. at Moscow State University, ${ }^{1}$ the cryogenic sapphire oscillator (CSO) project at the University of Western Australia (UWA) resulted in the creation of microwave signal sources with short-term frequency stability unmatched by any other type of electromagnetic oscillators ${ }^{2}$ including laser-cooled atomic fountain clocks.

The main drive to develop low-noise microwave oscillators in the mid-1980s was the quest to detect gravitational waves generated by supernovas. ${ }^{3}$ At the heart of the UWA, gravitational wave detection system was a cryogenically cooled niobium bar antenna, the vibration state of which was continuously monitored by a microwave readout system featuring a re-entrant cavity displacement transducer. ${ }^{4}$ To ensure the highest sensitivity for the microwave readout system, a low-phase-noise microwave oscillator was required to excite the transducer. ${ }^{5}$ Such an oscillator was constructed by utilizing the effect of the anomalously low dissipation of microwaves in cryogenically cooled sapphire dielectric., ${ }^{2,6,7}$

In parallel to the gravitational wave project, and beginning soon after, was the CSO project. Its goal was to create a stable frequency source relying on the existence of a turning point in the frequency-temperature dependence of the sapphire dielectric near $4 \mathrm{~K}$, annulling the effects of extrinsic temperature fluctuations. The exceptional stability of the microwave signals produced by CSOs found applications beyond the area of gravitational wave detection. Early applications included long baseline interferometry ${ }^{8}$ and frequency metrology with cold ion/atom clocks. For example, the National Measurement Institute (NMI), Sydney, has relied on a UWA CSOs as a fly-wheel oscillator for a laser-cooled ytterbium ion clock since the early 1990s. ${ }^{9,10}$

The first generation CSOs exhibited a fractionalfrequency stability close to one part in $10^{14}$ over integration times of $10-300 \mathrm{ss}^{6}$ This was improved by more than an order of magnitude by the mid-1990s when the noise mecha- nisms affecting the CSOs became better understood. ${ }^{7}$ The improvements in the CSO's frequency stability were assisted by the technological advances in growing ultrahigh-purity single-crystal sapphire via the heat exchange method HEMEX,${ }^{11}$ the availability of low-noise microwave components, and the cryogenic operation of the vital components of the oscillator. This oscillator was the first to reach a fractional-frequency stability less than one part in $10^{15}$ over integration times of $10-100 \mathrm{~s}$.

In 2000, a UWA sapphire oscillator was used at Observatoire de Paris for the development of a laser-cooled cesium-fountain clock. These experiments enabled the first experimental observation of the quantum projection noise in atomic-fountain clocks. ${ }^{12}$ In 2003, researchers at UWA and the Paris Observatory teamed up to undertake a search for the possible violations of Lorentz invariance by comparing the frequency of that CSO to that of a hydrogen maser. ${ }^{13}$ Also in 2003, another CSO was delivered to European Space Agency (CNES) to assist with the ground tests of the coldatom space clock "PHARAO" intended to operate in a microgravity environment. ${ }^{14}$ In 2005, at UWA a novel type of Michelson-Morley experiment involving two corotating CSOs was implemented to test the Lorentz invariance principle under the standard model extension framework and put the best limits on several Lorentz violating parameters. ${ }^{15,16}$

Between 2004 and 2006, four additional CSOs were developed at UWA. Two were built for the National Metrology Institute of Japan (NMIJ) at AIST, Tsukuba, ${ }^{17,18}$ one for the National Institute for Information and Communication Technology (NICT) in Tokyo, ${ }^{19}$ and the other to remain at UWA for the Frequency Standards and Metrology group. The fractional-frequency fluctuations of those oscillators are presently state-of-the-art approaching $5 \times 10^{-16}$ at an integration time of $20 \mathrm{~s}$ and highly reproducible over long measurement time periods. ${ }^{20}$ Their phase noise is $-85 \mathrm{dBc} / \mathrm{Hz}$ at $1 \mathrm{~Hz}$ on a $10 \mathrm{GHz}$ carrier (see Fig. 3 of Ref. 18). 
Over the past 15 years, in an effort to reduce the running cost of CSOs, various techniques have been tried to raise the temperature at which the resonator has a frequencytemperature turnover point. ${ }^{21-24}$ Researchers at JPL (Refs. 25 and 26) used thermomechanically compensated sapphire resonators and achieved fractional-frequency instabilities less than $1 \times 10^{-13}$ operating at $85 \mathrm{~K}$. Further research at JPL utilized the advances in cryocooler technology, resulting in frequency instability of $2 \times 10^{-14}$ at $1 \mathrm{~s}$ in a CSO at $10 \mathrm{~K}^{27}$ This CSO has potential for uninterrupted long term operation and avoids the need for costly cryogens.

At UWA, we have investigated increasing the fraction of impurities in sapphire to raise the compensation point ${ }^{28}$ to solid nitrogen temperatures $(50 \mathrm{~K})$. Such oscillators (having increased losses in the doped resonator and operating at an elevated temperature) could never rival the noise performance of liquid-helium-cooled sapphire oscillators. ${ }^{29}$

For some years, it has been expected that the fractionalfrequency stability of CSOs would soon be superseded by that of ultrastable lasers based on optical cavities with very high finesse and extremely low sensitivities to temperature and vibration. ${ }^{30-32}$ However, as was recently discovered, ${ }^{33}$ it is the thermal fluctuations in the mirror substrates of the optical cavities that impose fundamental limitations on their short-term frequency stability. Consequentially, CSOs are likely to retain their leading position in the ultrastable oscillator race until cryogenically cooled optical resonators become a reality.

This paper reviews the properties of cryogenically cooled sapphire-loaded-cavity (SLC) resonators, the structure of the CSOs, the principles of their operation, and their noise performance. The main focus of the current work is on design techniques, which enable optimal tuning and improved frequency stability. It brings together the expertise we have acquired while working with the CSOs over the past few years.

\section{THE RESONATOR}

At the heart of any CSO is a cylindrical sapphiredielectric resonator with its rotational axis aligned with the crystal axis. Eigenmodes of such a resonator are hybrid and feature all six components of the electromagnetic field. From a broad continuum of the eigenmodes, only two families of quasi-TM and quasi-TE modes are of practical importance to the design of ultrastable oscillators. These modes exhibit the highest field confinement to the dielectric and hence the highest electrical $Q$-factors. They are characterized by a small number of field variations along the axial and radial directions and a large number of field maxima around the azimuth, which is typical for modes characterized as 'whispering gallery' (WG), similar to acoustic modes observed by Rayleigh in St. Paul's Cathedral in London. ${ }^{34}$

A WG mode in a cylindrical dielectric resonator is a standing wave formed by a pair of traveling waves counterpropagating along the inside circumference of the dielectric cylinder and experiencing total internal reflection at the dielectric/air interface. Small geometrical imperfections of the sapphire cylinder, the presence of the coupling probes, as well as the misalignment of the crystal and rotation axes remove the degeneracy between the traveling wave "companions" and split the modes of resonance into two spectral components (doublets) having different coupling coefficients with frequencies separated by a few kilohertz. This effect is easily observed at cryogenic temperatures, where the frequency separation between the doublets can be a few hundred times larger than their individual resonance linewidths. A competition between doublets has never been observed in a loop oscillator due to the nonlinearity of its gain stage. Oscillations always establish at the frequency of one of the doublets (with the lower insertion loss), which saturate the loop microwave amplifier.

Enclosing the sapphire cylinder in a metal cavity protects it against the environmental perturbations and, most importantly for cryogenic applications, against surface contamination. This also reduces the required size and cost of the sapphire crystal relative to the size and cost of an openspace sapphire resonator tuned to the same frequency. One drawback of the SLC resonator is the relatively high density of spurious cavitylike modes, which may interact with the operational mode reactively or resistively. ${ }^{35,36}$ This problem can be solved by cutting a few radial and azimuthal slots in the cavity lids to intercept the flow of microwave currents and cause radiative loss of energy of the spurious modes. ${ }^{37}$ The $Q$-factors of the fundamental WG modes are not affected by the radiative slots, as their energy is almost entirely confined to the interior of the dielectric.

In the absence of crystal defects and surface contamination, the $Q$-factors of the WG modes in SLC resonators are limited by the sapphire-dielectric loss tangent $(\tan \delta)$. At temperatures $50<T<80 \mathrm{~K}$, the loss tangent is a very strong function of temperature, i.e., $\tan \delta \propto T^{5}$. This trend, however, does not hold at lower temperatures where crystal imperfections reduce the temperature dependence of the microwave losses. Even the highest quality sapphire crystals grown by the HEMEX method ${ }^{11}$ exhibit $\tan \delta \propto T$ over the temperature range of $2-12 \mathrm{~K}$. Nonetheless, the $Q$-factors measured in the liquid-helium-cooled HEMEX-sapphire resonators at frequencies around $10 \mathrm{GHz}$ are extremely high, of the order of $10^{9}$ at $T \approx 4.2 \mathrm{~K}$, with the highest measured $Q$-factor of $1.8 \times 10^{10}$ at $1.8 \mathrm{~K}^{38}$

Even the highest quality HEMEX sapphires are not free from paramagnetic impurities such as $\mathrm{Cr}^{3+},{ }^{39} \mathrm{Fe}^{3+},{ }^{40} \mathrm{Ti}^{3+}$, or $\mathrm{Mo}^{3+}$ ions. ${ }^{7}$ In such resonators, the paramagnetic ions, with only a small measurable effect on the $Q$-factor, strongly influence the frequency-temperature dependence of the WG modes by introducing a magnetic susceptibility to the sapphire crystal. The sign of the frequency shift resulting from the temperature induced change in the magnetic susceptibility is opposite to the frequency shift resulting from the temperature dependence of the sapphire-dielectric permittivity. With the magnitudes of the competing frequency shifts becoming comparable below $15 \mathrm{~K}$, frequency-temperature compensation is observed in SLC resonators. Such phenomena exist in all WG modes in HEMEX resonators at frequencies below the electron spin resonance frequency, which for $\mathrm{Mo}^{3+}$ ions is equal to $165 \mathrm{GHz}$. 


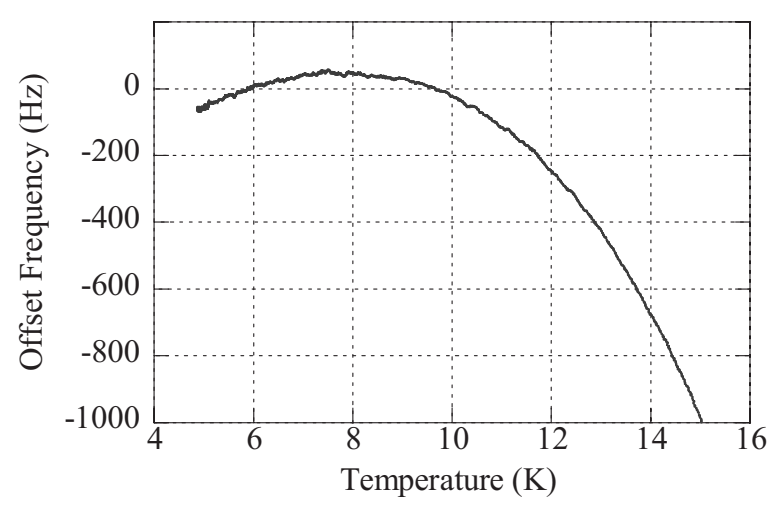

FIG. 1. Frequency-temperature dependence of an $11.9 \mathrm{GHz}$ WGE mode, measured by beating against another CSO and logging the difference frequency as the temperature of one sapphire resonator is ramped.

Figure 1 shows the frequency-temperature dependence of a $11.9 \mathrm{GHz}$ SLC resonator excited in the fundamental whispering gallery transverse electric (WGE) mode with azimuthal number $m=14$ (i.e., 28 field maxima in the azimuthal direction). It is this characteristic that defines these slightly doped sapphire samples as an excellent choice on which to base ultrastable oscillators. As the exact location of the frequency-temperature maxima depends on the concentration of paramagnetic impurities, their species type, and the nature of the mode excited, some HEMEX sapphires are unsuitable for application to a CSO, having a turning-point temperature either too high or too low. Also, the flatness (or curvature) of the frequency-temperature maximum in the vicinity of the turning point is an important parameter of the resonator, as it determines the required precision of the resonator temperature control. It is defined as $\kappa=\left(1 / f_{0}\right) d^{2} f / d T^{2}$, where $d^{2} f / d T^{2}$ is the second derivative of the resonance frequency as a function of temperature and $f_{0}$ is the mean resonance frequency. In HEMEX-crystal resonators, the smallest values of the fractional curvature are measured around $\kappa$ $\approx 10^{-9} \mathrm{~K}^{-2}$. This can be translated into a resonator fractional-frequency stability of order of $10^{-16}$, provided that the crystal temperature is kept within $1 \mathrm{mK}$ of the turning point and the temperature fluctuations are controlled to be less than $0.1 \mathrm{mK}$. These conditions are not difficult to meet for liquid-helium-cooled resonators due to the availability of high-sensitivity, yet low-noise, carbon-glass temperature sensors.

Figure 2 illustrates a typical approach to the temperature stabilization of a cryogenic SLC resonator. Here, the sapphire crystal is thermally insulated from the liquid helium bath by two evacuated stainless-steel vacuum cans. The temperature of the SLC resonator is stabilized to a precision of about $10 \mu \mathrm{K}$ by a feedback control system based on a temperature bridge connected to a four-terminal carbon-glass thermometer. The latter is embedded into the copper mounting shaft, to which the SLC resonator is directly attached. The top end of the copper shaft is connected to a stainlesssteel rod to ensure effective low-pass filtering of thermal fluctuations resulting from the boiling liquid helium. The outer can houses multiple microwave isolators which are needed to minimize the SLC resonator frequency pulling effect induced by the coaxial cable line resonances. Amplitude

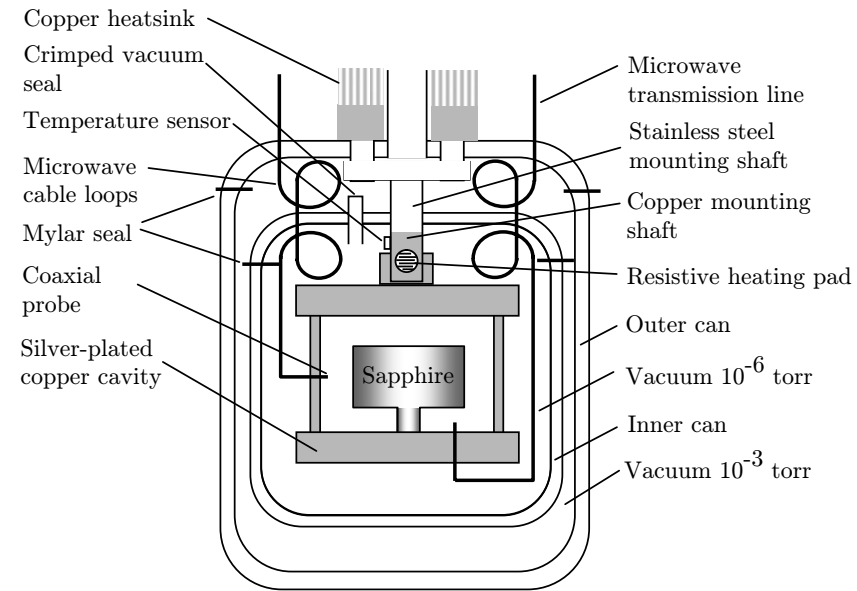

FIG. 2. Schematic diagram showing design features of the lower portion of the helium Dewar insert comprising two vacuum cans and the sapphire loaded cavity.

detectors, used in both the frequency and power control systems, are also located inside the outer can in the cryogenic environment to improve the oscillator's long-term frequency stability.

The SLC resonator in Fig. 2 is a single-spindle singlecrystal sapphire cylinder clamped from below. This type of clamping was suggested in Ref. 38 to reduce mechanical stress on the active region of the sapphire resonator where most of the electromagnetic field is confined. Such stress was found to be responsible for the frequency drift of the first generation CSOs utilizing double-spindle sapphire cylinders squeezed between the lids of the metal cavity. The latest resonators have also a top (though shorter) spindle, which is not clamped but used only to facilitate the cleaning process. ${ }^{20}$

Three major noise mechanisms limit the frequency stability of the CSO when it operates at the frequencytemperature turning point of the SLC resonator. The first noise mechanism is related to the intrinsic fluctuations of the Pound frequency discriminator. ${ }^{41,42}$ The second noise mechanism is associated with AM-index fluctuations of the microwave interrogation signal incident on the resonator. The third noise mechanism is related to fluctuations of dissipated microwave power in the sapphire resonator due to radiation pressure induced expansion in a solid dielectric resonator. ${ }^{43}$ The effect of the first two noise mechanisms on the spectral purity of the oscillator's signal can be greatly reduced by setting the SLC primary coupling close to critical. Such tuning, however, may require a few iterations (cool down cycles) due to the errors associated with the measurements of very small coupling coefficients and the strong dependence, near $4.2 \mathrm{~K}$, of sapphire $Q$-factor on its surface cleanliness.

In practice, the coupling must be set close to $10^{-4}$ at room temperature in order to obtain critical coupling at liquid-helium temperature. To measure such small coupling coefficients, we developed a new measurement technique involving frequency modulation of the microwave signal incident on the resonator with the subsequent synchronous demodulation of the reflected signal. ${ }^{44}$ The immunity of synchronous demodulation to the technical noise sources 


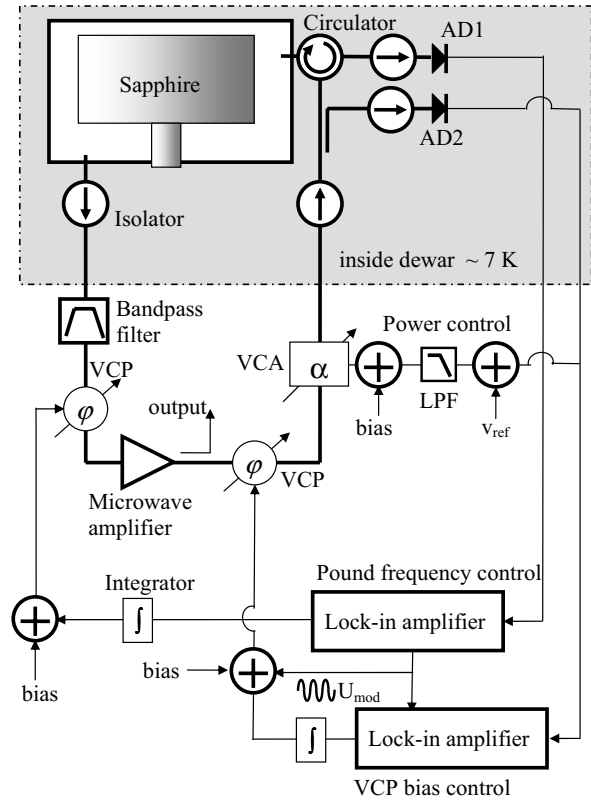

FIG. 3. Schematic diagram of a CSO showing three control systems stabilizing its frequency and power and eliminating the spurious amplitude modulation of the interrogation signal. Thick lines indicate microwave coaxial cables. $\mathrm{VCP}=$ voltage controlled pahse shifter, $\mathrm{VCA}=$ voltage controlled attenuator, $\mathrm{LPF}=$ low pass filter.

permits high fidelity measurements of coupling coefficients with a resolution unattainable by microwave network analyzers. We estimate that the smallest coupling coefficient which can be measured with the frequency modulation technique at a confidence level of $99.7 \%(3 \sigma)$ is approximately $5 \times 10^{-6}$.

In principle, microwave oscillators can be constructed at relatively high cryogenic temperatures $(40-80 \mathrm{~K})$ by making use of the simultaneous excitation of both whispering gallery transverse magnetic (WGH) and transverse electric (WGE) modes in the same SLC resonator. ${ }^{21,22}$ The anisotropy of sapphire permits the cancellation of the temperature dependence of the difference frequency between the two modes when the ratio of $\mathrm{WGE}$ and $\mathrm{WGH}$ mode resonance frequencies $f_{\mathrm{WGE}} / f_{\mathrm{WGH}} \approx \alpha_{\|} / \alpha_{\perp}$, where $\alpha_{\|}$and $\alpha_{\perp}$ are fractional temperature coefficients of sapphire-dielectric permittivity parallel and perpendicular to the crystal axis, respectively. For $X$-band SLC resonators cooled with liquid nitrogen $\alpha_{\|} / \alpha_{\perp} \approx 1.34$, which is equivalent to a mode separation of 3-4 GHz. The fractional-frequency stability of such a dual-mode oscillator operating at $40-50 \mathrm{~K}$ has been measured to be $4 \times 10^{-14}$ over an integration time of $1 \mathrm{~s} .^{45}$ This is adequate as a flywheel pump oscillator for a lasercooled cesium-fountain clock to achieve quantum limited performance. ${ }^{12}$ This makes dual-mode oscillators a viable alternative to the more expensive-running-cost liquid-heliumcooled oscillators for a number of frequency metrology applications and various long-term physics experiments in space.

\section{THE OSCILLATOR}

Figure 3 shows a schematic diagram of a frequency stabilized cryogenic oscillator. It is based on a high- $Q$ microwave SLC resonator which serves both as a bandpass filter of a self-sustaining loop oscillator and a dispersive element of a Pound frequency discriminator (FD). Operation of the Pound FD is based on rapid phase modulation (PM) of the incident microwave signal. If the oscillator frequency is different from that of the high- $Q$ resonator the signal reflected from the resonator acquires amplitude modulation (AM), the depth and the phase of which depend on the relative detuning between the oscillator and the resonator. By extracting the complex amplitude of the reflected signal (with an envelope detector) and comparing it to the original modulation signal (inside a lock-in amplifier as shown), an error voltage synchronously varying with the oscillator frequency is produced. The filtered error voltage is then applied to the voltage controlled phase (VCP) shifter in the loop oscillator steering its frequency to the chosen resonant mode of the high- $Q$ resonator. The frequency discriminator, filter, and VCP form a closed loop frequency control system which detects and cancels (within its bandwidth) fluctuations of the oscillator frequency from the cavity resonance frequency. For a frequency control loop with the sufficiently high gain, the quality of oscillator frequency stabilization is entirely determined by the noise properties of the frequency discriminator.

To ensure a reliable and efficient operation of the Pound frequency control system, the frequency of phase modulation must be correctly chosen. First of all, it should exceed the bandwidth of the high- $Q$ resonator. This minimizes the loss of the modulation sidebands inside the resonator and improves the discriminator's frequency-to-voltage conversion efficiency. This also eliminates the spurious zero crossings in the dependence of the error voltage on frequency. Such zero crossings may "confuse" the control system into locking the oscillator at a frequency far from the cavity resonance, which would be accompanied by enhanced phase noise due to the reduced value of the discriminator conversion efficiency. Another important factor which affects the choice of the modulation frequency is related to the noise properties of the Pound FD. The latter can be studied by replacing the resonator with an equivalent nondispersive load and measuring the spectrum of the voltage noise at the output of the lock-in amplifier at the same operating level of incident power. The resulting noise spectrum would consist of both "white" noise and flicker noise regions. The flicker noise has a technical origin and is mainly induced by the microwave carrier incident on the envelope detector. The spectral density of the flicker noise depends on the type of the envelope detector used, as well as the level of incident power. For a typical Pound FD operating at $X$-band, flicker noise dominates at Fourier frequencies below a few kilohertz. To avoid the degradation of the spectral resolution of the Pound discriminator by this flicker noise, the modulation frequency must be chosen well outside the flicker noise region.

The microwave power incident on the cryogenic sapphire resonator needs to be stabilized in order to minimize power induced frequency shifts associated with the radiation pressure effect. The magnitude of this effect increases with the $Q$-factor of the resonator, and for high quality HEMEX sapphire resonators (with $Q$-factors around $10^{9}$ ), it has been measured to be about $5 \times 10^{-11} / \mathrm{mW}^{43}$ 


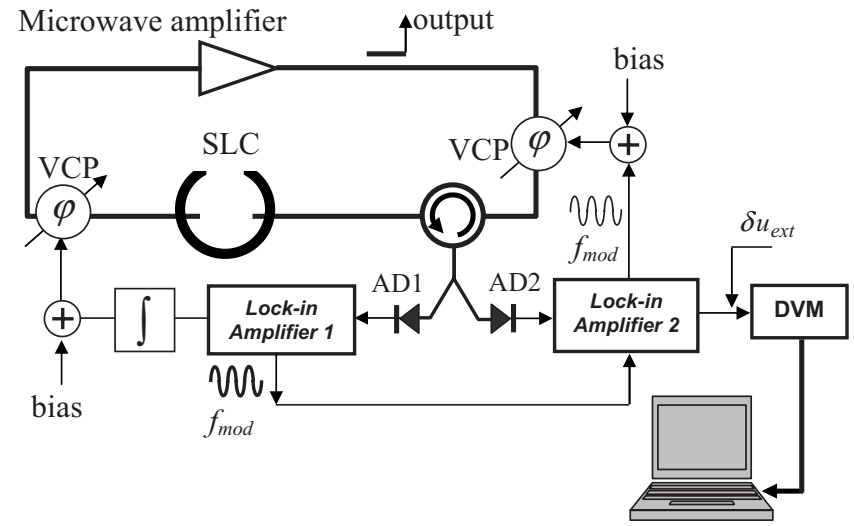

FIG. 4. Schematic diagram of a CSO with an external Pound FD illustrating the technique used for evaluation of the clock's frequency stability due to intrinsic fluctuations in the frequency discriminator.

The largest variations of microwave power incident on the SLC resonator are due to the gradual boil off of liquid helium, which changes the loss in the transmission lines connecting the cryogenic resonator to the oscillation sustaining stages. The power control system of a CSO is based on a cryogenic amplitude detector placed as close as possible to the sapphire resonator and a voltage controlled attenuator (VCA) located in the room temperature part of the loop oscillator next to the phase modulator (see Fig. 3).

Phase modulation of the microwave interrogating signal is often accompanied by spurious amplitude modulation due to the dependence of the VCP insertion loss on applied voltage. The AM sidebands in the spectrum of the interrogation signal are synchronously demodulated by the electronics of the Pound FD, producing an error voltage proportional to the real part of the SLC reflection coefficient, as opposed to the demodulation product of the rapid phase modulation (PM) sidebands, which varies as the imaginary part of the reflection coefficient. As a result, the error signal of the Pound FD changes its sign at a frequency which is shifted from the SLC resonant mode frequency. This means that any noise mechanism affecting the spurious AM index will also affect the frequency stability of the CSO. In the following sections, we analyze the influence of spurious AM on the frequency stability of the CSO as well as techniques minimizing such influences.

Finally, a temperature control system maintains the resonator temperature near the frequency-temperature turning point to ensure a first order immunity of the resonance frequency to ambient temperature fluctuations (see Fig. 1).

\section{A. Frequency stability limit due to intrinsic fluctuations of the Pound frequency discriminator}

Noise mechanisms affecting the frequency stability of the CSO can be divided into two groups. The first group directly affects the SLC resonance frequency. These include fluctuations of the ambient temperature, vibration, and fluctuations of dissipated microwave power. The second group of noise sources affects the quality of oscillator frequency stabilization. They are associated with the technical fluctuations in the electronics of the various feedback control

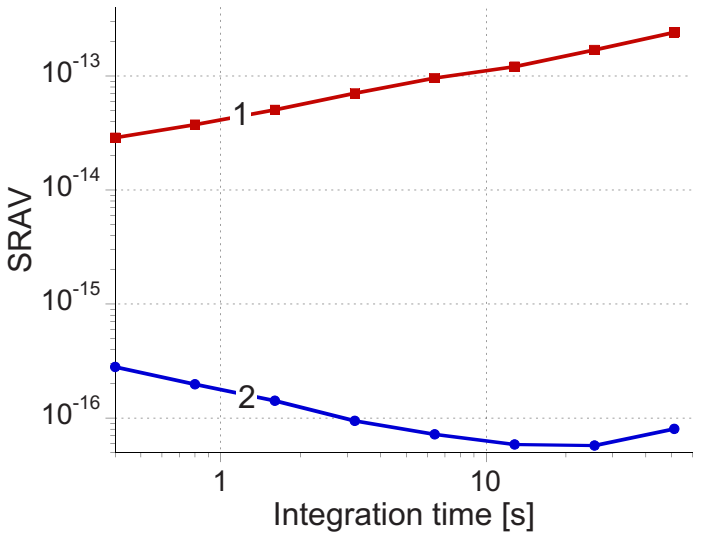

FIG. 5. (Color online) Curve 1 is the Allan frequency deviation of a freerunning oscillator. Curve 2 is the FD noise floor calculated from voltage noise measurements.

circuits mentioned above. The effect of the second group of noise mechanisms on frequency stability of the CSO is discussed below.

An estimate of the frequency instability induced by intrinsic fluctuations of the Pound FD can be obtained by making use of an additional FD, external with respect to the primary frequency control loop (see Fig. 4).

The voltage noise $\left(\delta u_{\text {ext }}\right)$ at the output of the external FD can be expressed as a symbolic sum,

$$
\delta u_{\mathrm{ext}}=S_{\mathrm{FD}} \delta f_{\mathrm{osc}} \oplus \xi,
$$

where $S_{\mathrm{FD}}$ is the frequency-to-voltage conversion coefficient of the external $\mathrm{FD}, \delta f_{\text {osc }}$ corresponds to frequency fluctuations of the locked oscillator, and $\xi$ denotes the intrinsic voltage fluctuations of the additional frequency discriminator. Noise processes $\delta f_{\text {osc }}$ and $\xi$ in Eq. (1) are independent.

Assuming that both FDs are identical and the gain of the frequency control system is high enough that frequency fluctuations of the free-running oscillator do not make any significant contribution to $\delta u_{\mathrm{ext}}$, Eq. (1) is simplified as

$$
\delta u_{\mathrm{ext}} \approx \sqrt{2} \xi \text {. }
$$

By combining Eqs. (1) and (2), the relationship between oscillator frequency fluctuations and intrinsic voltage noise at the output of the external FD is obtained,

$$
\delta f_{\mathrm{osc}}=\frac{\delta u_{\mathrm{ext}}}{\sqrt{2} S_{\mathrm{FD}}} .
$$

Characterizing the oscillator frequency fluctuations in terms of its Allan deviation $\sigma_{y}(\tau)$ and making use of Eq. (3) result in

$$
\sigma_{y}(\tau)=\frac{\sigma_{u}(\tau)}{\sqrt{2} S_{\mathrm{FD}} f_{0}},
$$

where $f_{0}$ is the mean frequency of oscillations, $\sigma_{u}(\tau)$ is the Allan deviation of voltage noise $\delta u_{\mathrm{ext}}$, and $\tau$ is the integration time.

The dependence of $\sigma_{y}(\tau)$ is shown in Fig. 5 (curve 2). This dependence, which is referred to below as a FD noise floor, was deduced from the measurements of voltage noise at the output of the external FD with the following set of parameters: the resonator unloaded $Q$-factor $=1.5 \times 10^{9}$, the 


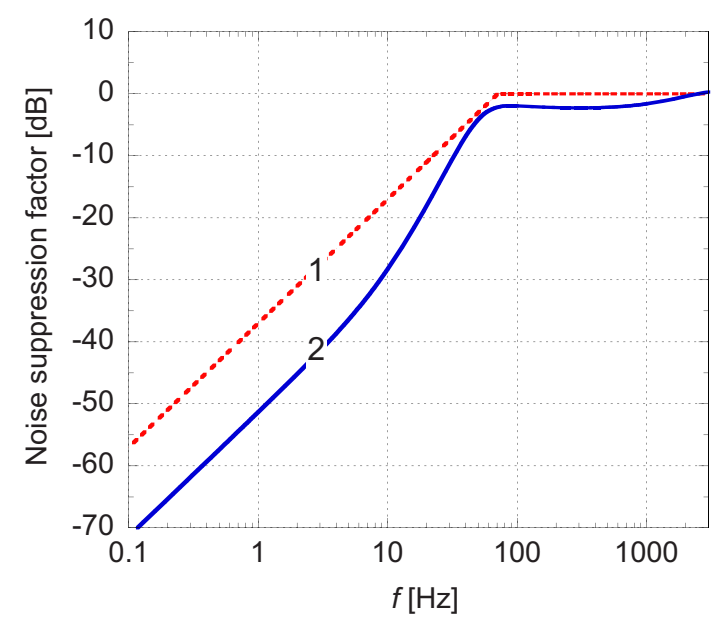

FIG. 6. (Color online) Noise suppression factor of the frequency control system curve 1: approximation obtained from the ratio of two phase noise spectra; curve 2: calculated dependence for the integrator based feedback loop.

primary coupling coefficient $\beta=1.06$, the power incident on the resonator $P_{\mathrm{inc}}=4 \mathrm{~mW}$, and the index of phase modulation $\varphi_{m}=0.03 \mathrm{rad}$.

For $\tau<30 \mathrm{~s}$, the FD noise floor improves with integration time as $\sigma_{y} \propto 10^{-16} / \sqrt{\tau}$. This limit is set by the white voltage fluctuations of the amplitude detector in the FD circuit. However, for $\tau>30 \mathrm{~s}$ the FD noise floor deteriorates as $\sigma_{y} \propto \sqrt{\tau}$, which has been found to be related to the voltage noise of the digital signal processing stage of the lock-in amplifier.

Curve 1 in Fig. 5 shows the Allan frequency deviation of a free-running oscillator $\sigma_{y}^{f / \text { run }}(\tau)$. The oscillator was based on a SLC resonator (with the above listed parameters) operating at its frequency-temperature turning point. The observed dependence $\left[\sigma_{y}^{f / \text { run }}(\tau) \approx 3 \times 10^{-14} \sqrt{\tau}\right]$ is typical of random-walk frequency noise. Such noise has been explained by temperature induced fluctuations of the time delay in the long transmission lines connecting the room temperature components of the loop oscillator with the cryogenic SLC resonator.

The data in Fig. 5 enable one to design a frequency control system with enough gain to suppress the frequency fluctuations of a free-running oscillator well below the Pound FD noise floor. This is done by converting the Allan deviations $\sigma_{y}^{f / r u n}(\tau)$ and $\sigma_{y}(\tau)$ into equivalent phase noise spectra. ${ }^{46,47}$ The respective spectral densities of phase fluctuations of the free-running oscillator and the frequency discriminator are $S_{\varphi}^{f / r u n}(f) \approx 10^{-8} / f^{4}$ and $S_{\varphi}^{\mathrm{FD}}(f) \approx 2 \times 10^{-12} / f^{2}$, where $f$ is the Fourier frequency.

The structure of the frequency control system can be determined by plotting the ratio of two phase noise spectra $R=S_{\varphi}^{\mathrm{FD}} / S_{\varphi}^{f / r u n}$ as a function of Fourier frequency (curve 1 in Fig. 6). This ratio characterizes the noise suppression factor of the feedback loop which is needed to suppress the frequency fluctuations of the free-running oscillator to the noise floor of the Pound FD. By using the dependence $R(f)$, one can determine the unity-gain bandwidth of the control system (about $70 \mathrm{~Hz}$ ) and the order of the loop filter required to

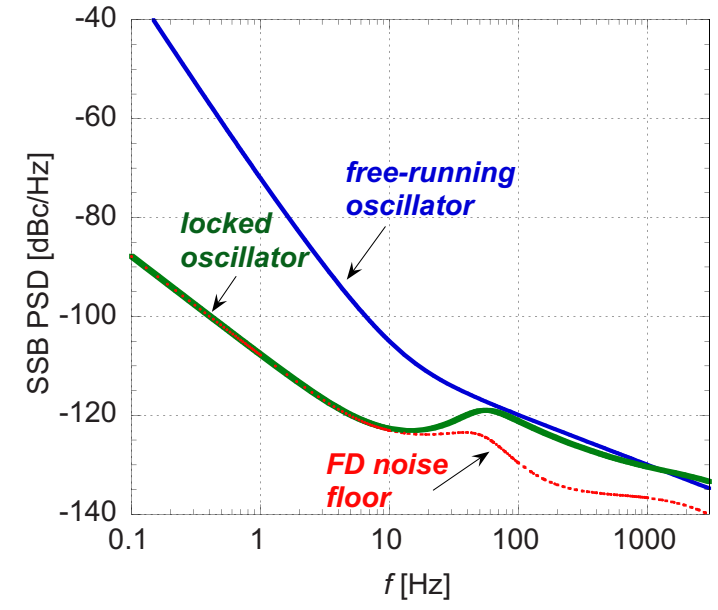

FIG. 7. (Color online) Calculated spectral densities of phase fluctuations of both a free-running and a frequency stabilized CSOs, along with the phase noise floor of the Pound FD.

cope with the quickly divergent phase noise spectrum of the free-running oscillator $S_{\varphi}^{f / r u n}$.

Curve 2 in Fig. 6 shows the calculated noise suppression factor of the frequency control system used on our CSOs. The simulations were conducted by assuming a loop filter consisting of a "leaking" integrator with a $100 \mathrm{~Hz}$ corner frequency and an active all-pass filter with corner frequencies of 10 and $100 \mathrm{~Hz}$. The latter ensured a stable operation of the frequency control system by compensating for the phase lag introduced by the digital lock-in amplifier. The low frequency gain of the control system was chosen to be higher than was empirically determined (curve 1) to avoid any limitations on oscillator frequency stability associated with the phase noise of a free-running oscillator.

The calculated phase noise spectra of both a free-running and a frequency stabilized sapphire oscillator are shown in Fig. 7. The main idea behind these calculations was to evaluate the phase noise of the CSO (which was not directly measured) and prove that it would not degrade outside the bandwidth of the frequency control loop. In principle, as it is seen from Fig. 7, the bandwidth of the frequency servo can be extended to a few kilohertz, if low phase noise operation of the CSO at higher Fourier frequencies is important.

The frequency-to-voltage conversion efficiency of the frequency discriminator $\left[S_{\mathrm{FD}}\right.$ in Eq. (4)] is a key parameter which determines the frequency stability of a microwave oscillator. For given parameters of a SLC resonator, $S_{\mathrm{FD}}$ is a function of both the power incident on the resonator $\left(P_{\text {inc }}\right)$ and the PM index $\left(\varphi_{m}\right)$. To find the optimal values of $P_{\text {inc }}$ and $\varphi_{m}$ at which the coefficient $S_{\mathrm{FD}}$ is maximized, one has to calculate the complex amplitude of the first harmonic at the frequency of modulation in the spectrum of the signal from the amplitude detector (AD). Because of the nonlinearity of the $\mathrm{AD}$ (as it is a square-law envelope detector), such calculations must be conducted in the time domain by following the steps described below.

Firstly, the instantaneous power of a signal incident on the $\mathrm{AD}$, where $\varphi_{m} \ll 1$, is determined from 


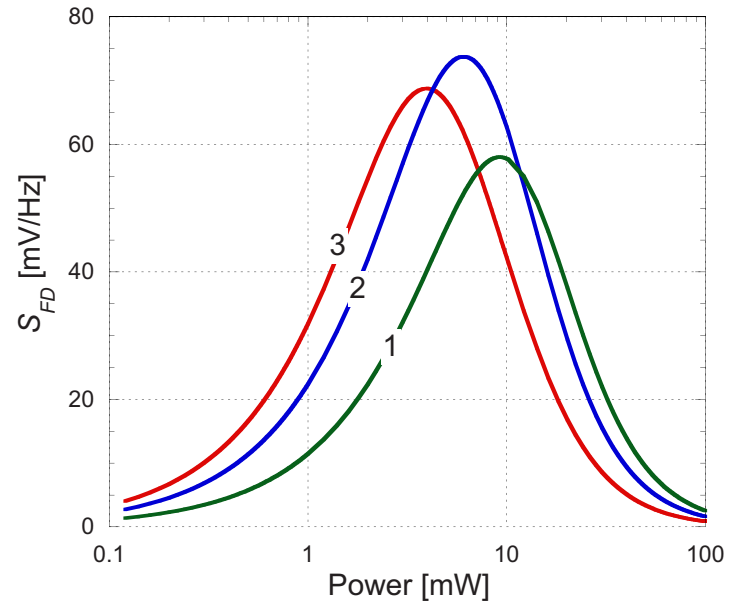

FIG. 8. (Color online) Frequency-to-voltage conversion efficiency of the Pound FD as a function of incident power: curve $1\left(\varphi_{m}=0.1\right)$, curve 2 $\left(\varphi_{m}=0.2\right)$, and curve $3\left(\varphi_{m}=0.3\right)$. The following list of parameters was assumed: $P_{\text {sat }}=0.5 \mathrm{~mW}, \gamma_{\mathrm{det}} \approx 750 \mathrm{mV} / \mathrm{kHz}, K_{\mathrm{LA}}=1$, a resonator coupling coefficient $\beta=0.7$, and a $Q$-factor of $8 \times 10^{8}$.

$$
P_{\text {det }}=P_{\text {inc }}\left|J_{0}\left(\varphi_{m}\right) \Gamma+j 2 J_{1}\left(\varphi_{m}\right) \cos \left(\omega_{\text {mod }} t\right)\right|^{2},
$$

where $J_{0}$ and $J_{1}$ are Bessel functions of the first kind, $\omega_{\bmod }$ $=2 \pi f_{\text {mod }}$ is the angular frequency of modulation, and $\Gamma$ is the resonator reflection coefficient. In deriving Eq. (5), it was assumed that the modulation frequency is much larger than the resonator bandwidth, i.e., $f_{\text {mod }} \gg \Delta f_{\text {res }}$.

The instantaneous voltage at the output of the $\mathrm{AD}$ is found by specifying the nonlinear response of the $\mathrm{AD}$, which, for the majority of microwave detectors, can be approximated by

$$
u_{\mathrm{det}}=\gamma_{\mathrm{det}} \frac{P_{\mathrm{det}}}{\sqrt{1+\left(P_{\mathrm{det}} / P_{\mathrm{sat}}\right)^{2}}},
$$

where $\gamma_{\text {det }}$ is the detector power-to-voltage conversion efficiency and $P_{\text {sat }}$ its saturation power. For a typical $X$-band detector used in our oscillators, $\gamma_{\text {det }} \approx 750 \mathrm{mV} / \mathrm{mW}$ and $P_{\text {sat }} \approx 0.5 \mathrm{~mW}$. By taking the fast Fourier transform of the detector signal $u_{\text {det }}$ and computing the complex amplitude of its first harmonic at the modulation frequency $U_{\text {det }}\left(f_{\text {mod }}\right)$, the parameter $S_{\mathrm{FD}}$ is calculated from

$$
S_{\mathrm{FD}}=K_{\mathrm{LA}} \frac{\Delta U_{\mathrm{det}}}{\Delta f},
$$

where $K_{\mathrm{LA}}$ is the gain of the lock-in amplifier and $\Delta U_{\mathrm{det}}$ is the variation of the amplitude of the first harmonic of the detector signal due to the change in oscillator frequency $\Delta f$ $=f_{\text {osc }}-f_{\text {res }}$. The results of such calculations are summarized in Fig. 8, which shows parameter $S_{\mathrm{FD}}$ as a function of incident microwave power at the different indexes of phase modulation.

The calculations were conducted with the oscillator mean frequency $f_{\text {osc }}$ equal to the cavity resonance frequency $f_{\text {res }}$, which corresponds to the normal operating conditions of a frequency locked oscillator. The resonator coupling coefficient was assumed to be $\beta=0.7$. As follows from the data in Fig. 8, the maximum FD sensitivity is achieved at $P_{\text {inc }}$ $=0.6 \mathrm{~mW}$ and $\varphi_{m} \approx 0.2 \mathrm{rad}$. The optimal index of phase modulation obtained is much smaller than one might expect

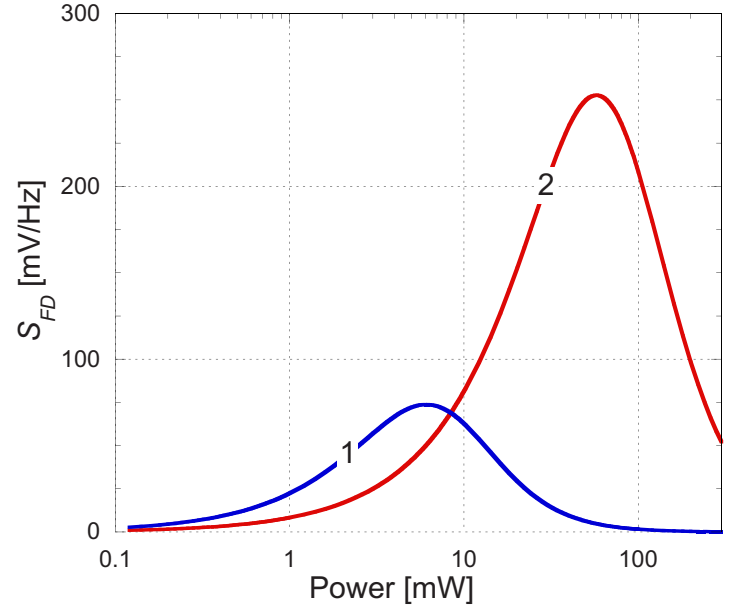

FIG. 9. (Color online) Optimized frequency-to-voltage conversion efficiency of the Pound FD as a function of incident power: curve $1(\beta=0.7$, $\left.\varphi_{m}=0.2\right)$, curve $2\left(\beta=0.9, \varphi_{m}=0.07\right)$.

from the linear analysis. If it was not for the nonlinearity of the $\mathrm{AD}$, an optimal value of the PM-index would be chosen near $1 \mathrm{rad}$, corresponding to the first maximum of the transcendental function $J_{0}(x) J_{1}(x)$.

The closer the resonator's coupling is to critical, the smaller the optimal PM-index at which the maximum FD sensitivity is achieved. For example, at $\beta=0.9$, the maximum value of $S_{\mathrm{FD}}$ is achieved at $P_{\mathrm{inc}}=56 \mathrm{~mW}$ and $\varphi_{m} \approx 0.07 \mathrm{rad}$. In such a case, the product $P_{\text {inc }} \varphi_{m}$ is 3.3 times larger than in the previously analyzed situation (where $\beta=0.7$ ), which directly translates in the enhancement of the FD sensitivity $S_{\mathrm{FD}}$ (see Fig. 9).

Setting the resonator's coupling close to critical not only improves the efficiency of the Pound FD but also allows one to minimize the oscillator frequency instability induced by fluctuations of the index of spurious amplitude modulation (see below). Unfortunately, achieving critical coupling at liquid helium temperatures is almost impossible due to the relatively large uncertainty in the $Q$-factor of the SLC resonator when cooling it from room temperature. One possible solution to the problem may involve the use of interferometric signal processing in order to suppress the microwave carrier incident on the $\mathrm{AD}$, as shown in Fig. 10.

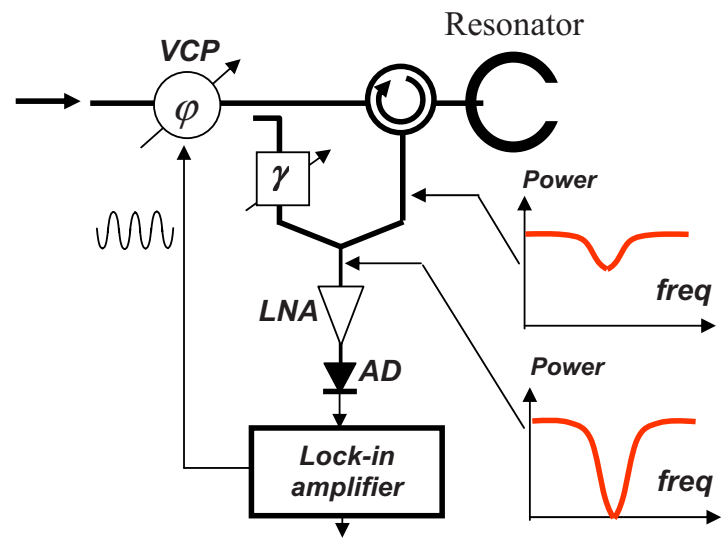

FIG. 10. (Color online) Schematic diagram of the interferometric Pound FD. 
Figure 10 shows a microwave Pound frequency discriminator with interferometric signal processing. The microwave signal reflected from the high- $Q$ resonator destructively interferes with a fraction of incident signal at the "dark port" of the interferometer. This cancels the carrier of the difference signal while preserving the modulation sidebands. In other words, a balanced microwave interferometer with a high- $Q$ resonator in one arm can be considered as an equivalent resonator having the same $Q$-factor and coupling equal to critical. Having suppressed the microwave carrier, one can think about immediately introducing a low-noise microwave amplifier after the interferometer's dark port in order to amplify the residual noise sidebands resulting from the loop oscillator frequency fluctuations. In such a case, one can expect an additional improvement in the FD sensitivity due to its reduced effective noise temperature.

A problem one will face while trying to build an interferometric Pound FD based on a cryogenic SLC resonator is the balancing of the cryogenic interferometer. This, we believe, could be solved by placing a stepper-motor driven mechanical attenuator/phase-shifter assembly in the interferometer's compensating arm. Another question, which is yet to be answered, is related to the frequency stability of the equivalent resonator. The point is that any phase variations in the compensation arm $\delta \psi$ result in variations of the resonance frequency of the equivalent resonator $\delta \tilde{f}_{\text {res }}$ in accordance with

$$
\delta \tilde{f}_{\text {res }}=\Delta f_{\text {res }} \frac{1-\beta^{2}}{2 \beta} \delta \psi \text {. }
$$

For a compact, cryogenically cooled microwave interferometer based on a strongly coupled SLC resonator ( $\beta$ $\approx 0.7 \cdots 0.9$ ), the above limitation may not be that serious, but this needs to be experimentally verified by measuring the spectral density (or Allan deviation) of the phase fluctuations $\delta \psi$.

\section{B. Frequency stability limit due to AM-index fluctuations of the interrogation signal}

As mentioned above, amplitude modulation sidebands in the spectrum of the interrogation signal shift the operating frequency of the CSO relative to the cavity resonance by

$$
\Delta f=\Delta f_{\text {res }} \frac{(1-\beta)^{2}}{2 \beta} \frac{m}{\varphi_{m}},
$$

where $m$ is the AM index of the interrogation signal, given by

$$
m=\frac{\dot{\alpha}}{2 \alpha} U_{m},
$$

where $U_{m}$ is the amplitude of the modulating signal with frequency $f_{\text {mod }}$. The parameters $\alpha$ and $\dot{\alpha}$ are the insertion loss of the phase modulator and its first voltage derivative, respectively. Both $\alpha$ and $\dot{\alpha}$ are functions of the VCP bias voltage $U_{0}$. Equation (9) characterizes the power-to-frequency conversion in the Pound FD. Due to this effect, the frequency stability of the CSO could suffer from spurious AM of the interrogation signal.
There are a few noise mechanisms responsible for amplitude fluctuations of the interrogation signal. One such mechanisms is related to the upconversion of the VCP bias voltage fluctuations $\left(\delta U_{0}\right)$. By making use of Eq. (10), the relationship between AM-index fluctuations of the interrogation signal $(\delta m)$, and the VCP bias voltage fluctuations $\left(\delta U_{0}\right)$, the following can be obtained:

$$
\delta m=\frac{U_{m}}{2 \alpha}\left(\ddot{\alpha}-\frac{\dot{\alpha}^{2}}{\alpha}\right) \delta U_{0},
$$

where $\ddot{\alpha}$ is the second voltage derivative of the VCP insertion loss.

Fluctuations of the amplitude $U_{m}$ of the modulating signal result in

$$
\delta m=\frac{\dot{\alpha}}{2 \alpha} \delta U_{m} .
$$

The ambient temperature fluctuations $\delta T$ give rise to AMindex fluctuations with magnitude

$$
\delta m=\delta U_{m} \frac{d}{d T}\left(\frac{\dot{\alpha}}{2 \alpha}\right) \delta T .
$$

If the insertion loss $\alpha$ is a nonmonotonic function of the bias voltage, AM-index fluctuations of the interrogating signal can be suppressed by operating the phase modulator at a turning point $\left(U_{0}=U_{\mathrm{TP}}\right)$, where $\dot{\alpha}=0$. This turning point can either be intrinsic to the VCP or it can be created by the injection of an appropriate compensation signal (derived from the same modulation signal) to another voltage controlled component placed in series with the given VCP. In this section, we will analyze the noise properties of the feedback control system which keeps the VCP bias voltage locked to the turning point.

A schematic diagram of the VCP bias control system is shown in Fig. 3. Here, a combination of a microwave amplitude detector (AD2) and lock-in amplifier acts as a voltage discriminator producing an error signal proportional to the first derivative of the insertion loss on the bias voltage. The error signal is filtered and superimposed on the VCP bias voltage $U_{0}$.

By denoting the total dc voltage applied to the VCP as $U_{\varphi}$, the characteristic equation of the bias control system can be written as

$$
U_{\varphi}=U_{0}+K_{F}(p) u_{\mathrm{LA}},
$$

where $K_{F}(p)$ is a differential operator of a low-pass filter in the control loop and $u_{\mathrm{LA}}$ is the lock-in amplifier output voltage, which, in general, is a nonlinear function of $U_{\varphi}$.

Linearizing Eq. (14) in the vicinity of the turning point $\left(U_{\varphi} \approx U_{T P}\right)$ results in the following expression for the fluctuations of the VCP bias voltage,

$$
\delta U_{\varphi}=\frac{\delta U_{0}}{1+\gamma} \ominus \frac{\gamma}{1+\gamma} \frac{\delta n}{S_{\mathrm{VD}}},
$$

where $\gamma$ is the gain of the feedback loop and $S_{\mathrm{VD}}$ is the conversion efficiency of the voltage discriminator given by 


$$
S_{\mathrm{VD}}=K_{\mathrm{LA}} \gamma_{\mathrm{det}} P_{\mathrm{det}} U_{m} \ddot{\alpha} / \alpha,
$$

where $K_{\mathrm{LA}}$ is the lock-in amplifier gain, $\gamma_{\mathrm{det}}$ is the amplitude detector conversion efficiency, and $P_{\text {det }}$ is the detected microwave power. The parameter $\delta n$ in Eq. (15) is the noise floor of the voltage discriminator. It reflects a joint contribution of power fluctuations of the interrogation signal $\left(\delta m_{\text {osc }}\right)$ and intrinsic fluctuations from the amplitude detector $\left(\xi_{\text {det }}\right)$ to the voltage noise at the output of the lock-in amplifier. Hence,

$$
\delta n \approx K_{\mathrm{LA}}\left\{2 \gamma_{\mathrm{det}} P_{\mathrm{det}} \delta m_{\mathrm{osc}} \oplus \xi_{\mathrm{det}}\right\} .
$$

The rms value of $\xi_{\text {det }}$ can be expressed as $k_{B} T_{\text {det }} \sqrt{1 \mathrm{~Hz}}$, where $k_{B}$ is Boltzmann's constant and $T_{\text {det }}$ is the effective noise temperature of the $\mathrm{AD}$ at the modulation frequency $f_{\text {mod. }}$.

By substituting Eqs. (15) and (17) in Eq. (11) and assuming that $U_{\varphi}=U_{\mathrm{TP}}(\dot{\alpha}=0)$, one can derive an analytical expression for the AM-index fluctuations of the interrogation signal, which are induced by the bias control circuit, as follows:

$$
\delta m_{\text {induced }} \approx \frac{U_{m}}{2 \alpha} \ddot{\alpha} \frac{\delta U_{0}}{1+\gamma} \oplus \frac{\gamma}{1+\gamma}\left\{\frac{k_{B} T_{\mathrm{det}}}{2 P_{\mathrm{det}}}-\delta m_{\mathrm{osc}}\right\} .
$$

Remembering that total amplitude fluctuations of the interrogation signal $\delta m=\delta m_{\mathrm{osc}}+\delta m_{\text {induced }}$ and assuming that $|\gamma| \gg 1$ result in

$$
\delta m=\frac{k_{B} T_{\mathrm{det}}}{2 P_{\mathrm{det}}} .
$$

This result indicates that the intrinsic noise of the microwave detector is the only factor which determines the level of amplitude fluctuations of the interrogation signal at $f_{\text {mod }}$.

By combining Eqs. (9) and (19), one can estimate the frequency stability of the CSO due to intrinsic noise of the bias control system from

$$
\sigma_{y}(\tau)=\frac{1}{2 Q \varphi_{m}} \frac{(1-\beta)^{2}}{2 \beta} \frac{k_{B} T_{\mathrm{det}}}{2 P_{\mathrm{det}} \sqrt{2 \tau}},
$$

where $Q$ is the SLC resonator unloaded $Q$-factor and $\tau$ is the integration time. For a typical set of parameters corresponding to the liquid-helium-cooled CSO, unloaded $Q \approx 8 \times 10^{8}$, $\beta \approx 0.7, \varphi_{m} \approx 0.05, T_{\mathrm{det}} \approx 10^{12} \mathrm{~K}$, and $P_{\mathrm{det}} \approx 0.3 \mathrm{~mW}$, from Eq. (20) one obtains $\sigma_{y}(\tau) \approx 1.3 \times 10^{-17} / \sqrt{\tau}$, which is 20 times less than the limit imposed by intrinsic fluctuations of the Pound FD.

In practice, the above limit (20) can be inferred by measuring the Allan deviation of voltage fluctuations $\widetilde{\sigma}_{u}(\tau)$ at the output of the lock-in amplifier of the VCP bias control system. These measurements must be performed with the control loop open and modulation signal switched off $\left(U_{m}=0\right)$, so that only the intrinsic fluctuations of the voltage discriminator are measured. The relationship between $\widetilde{\sigma}_{u}(\tau)$ and oscillator fractional-frequency instability is given by

$$
\sigma_{y}(\tau) \approx \frac{1}{2 Q \varphi_{m}} \frac{(1-\beta)^{2}}{2 \beta} \frac{\widetilde{\sigma}_{u}(\tau)}{2 U_{\mathrm{det}} K_{\mathrm{LA}}},
$$

where $U_{\text {det }}$ is the dc voltage produced by the amplitude detector (AD2 in Fig. 3) of the bias control system.

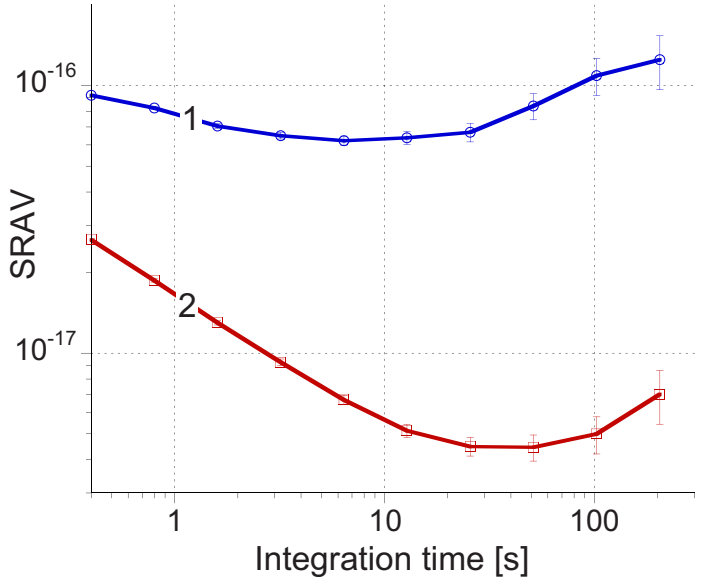

FIG. 11. (Color online) Inferred fractional-frequency stability from voltage fluctuations due to amplitude modulation of the interrogation signal. Curve 1: modulation signal on (bias control loop open). Curve 2: modulation signal off (bias control loop open).

By having measured $\widetilde{\sigma}_{u}(\tau)$, we used Eq. (21) in order to infer the frequency stability of the CSO due to the spurious amplitude modulation of the interrogation signal. The inferred dependence of $\sigma_{y}(\tau)$ is shown by curve 2 in Fig. 11. It was calculated with the same set of oscillator parameters used earlier when evaluating the theoretical limit (20). Both the inferred result and the predicted theoretical limit are in good agreement for the integration times less than $10 \mathrm{~s}$. The divergence between the inferred and predicted results for $\tau>20 \mathrm{~s}$ is due to the $1 / f$-noise of the lock-in amplifier, which was not taken into account when deriving Eq. (20).

By repeating the above measurements with the modulation signal turned on (the bias control loop still open) and making use of Eq. (21), we evaluated the oscillator's frequency stability due to the combined effect of all noise sources contributing to AM-index fluctuations of the interrogation signal. This is shown by curve 1 (Fig. 11) which passes below $9 \times 10^{-17}$ over the range of integration times of $0.3-30 \mathrm{~s}$. Such a relatively low weak influence of the spurious AM on the oscillator's frequency stability could be explained by the near critical coupling to the SLC resonator and the use of a VCP with a very flat dependence of the insertion loss on bias voltage in Eqs. (20) and (21).

In the general case of a weakly coupled resonator or a VCP with a nonmonotonic dependence of insertion loss on voltage, the spurious $\mathrm{AM}$ of the interrogation signal may seriously limit the frequency stability of the CSO. This problem can be resolved by injecting a compensation signal (derived from the same modulation signal applied to the $\mathrm{VCP}$ ) to the VCA of the power control system (see Fig. 3). To keep the spurious AM suppressed, one can either adjust the amplitude and phase of the compensation signal or vary the VCP bias voltage, which could be "entrusted" to the corresponding feedback control system.

In addition to inferring the oscillator frequency stability from Eq. (21), one can deduce it from in situ measurements by involving the use of an external voltage discriminator. A schematic diagram of such measurements is shown in Fig. 12. The idea of this approach is identical to that used for the evaluation of Pound FD noise floor, which means that Eq. (4) 


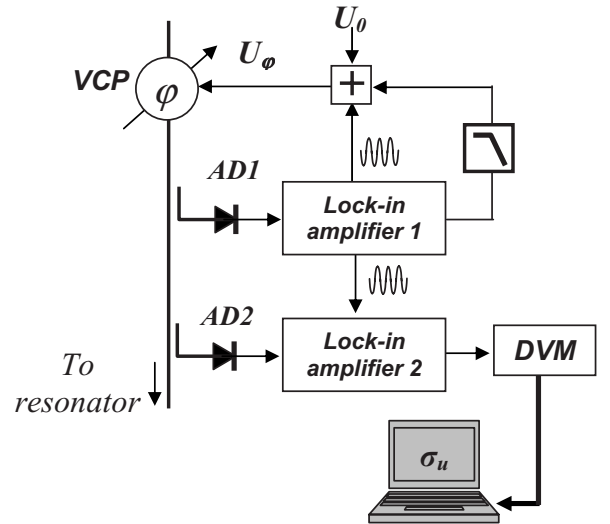

FIG. 12. Experimental setup for the in situ evaluation of the frequency stability of the CSO due to amplitude fluctuations of the interrogation signal. The digital voltmeter (DVM) and a personal computer are used to compute the square root Allan variance (SRAV) of fractional voltage fluctuations at the output of the additional channel of the measurement system.

with $S_{\mathrm{FD}}$ replaced with $S_{\mathrm{VD}}$ can be applied to convert the Allan voltage deviation into the oscillator's frequency instability.

Finally, it is worth mentioning that the semiexperimental approach to the evaluation of the oscillator frequency stability based on the relationship between $\widetilde{\sigma}_{u}(\tau)$ and $\sigma_{y}(\tau)$ in Eq. (21) can be used to determine the optimal choice of VCP bias control circuit in a way similar to that which was described earlier in relation to the frequency control system.

\section{Frequency stability of the CSOs}

Two almost identical CSOs operating at $11.2 \mathrm{GHz}$ were constructed to measure their short-term frequency stability. In each oscillator, the temperature of the SLC resonator was maintained at its frequency-temperature turnover point (near $7 \mathrm{~K}$ ) with a commercial temperature controller by using a carbon glass thermometer as a temperature sensor. The beat frequency between the two oscillators $\left(f_{\text {beat }}=131.181 \mathrm{kHz}\right)$ was measured with a frequency counter referenced to a $10 \mathrm{MHz}$ signal from a hydrogen maser. The fractionalfrequency uncertainty of the measurement system was limited by the countertriggering errors and is a diminishing function of the integration time: $\sigma_{y}^{\text {count }} \approx 10^{-16} / \sqrt{\tau}$.

Figures 13 and 14 show the Allan deviation of the fractional-frequency fluctuations of an individual CSO $\left(\sigma_{y}\right)$ as a function of integration time. It nonmonotonically behaves over the range of integration times $3<\tau<1000 \mathrm{~s}$ reaching a minimum $\sigma_{y}^{\mathrm{min}} \approx 5.6 \times 10^{-16}$ at $\tau \approx 20 \mathrm{~s}$ and remaining below the level of $10^{-15}$. This frequency stability is the state of the art for modern-day microwave signal sources.

In addition to intercomparison of two CSOs, we also compared the "beat" between the CSO and an atomic clock. In the latter case, we used a microwave frequency synthesizer phase referenced to a hydrogen maser and operating near $11.2 \mathrm{GHz}$. The results of this comparison are given by curves 1 and 2 in Fig. 13. As expected, the maser noise dominates the noise measurements at the relatively short integration times $(\tau<2000 \mathrm{~s})$, while at larger integration times, the results of both experiments very well agree with each other. The data in Fig. 13 indicate that the long-term

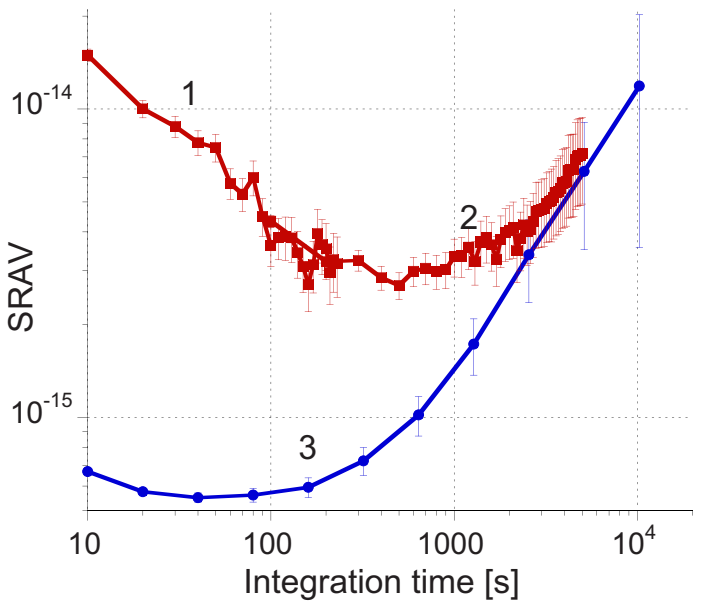

FIG. 13. (Color online) Measured Allan deviation for a single oscillator from the beat between two nominally identical cryogenic sapphire oscillators (curve 3: gate time of $10 \mathrm{~s}$ ) and between one cryogenic sapphire oscillator and a hydrogen maser (curve 1: gate time of $10 \mathrm{~s}$; curve 2: gate time of $100 \mathrm{~s}$ ) to determine the long-term performance.

behavior of the CSO $\left(\sigma_{y} \propto \sqrt{\tau}\right)$ is limited by the random walk of its frequency, which is, most likely, caused by the ambient temperature fluctuations influencing the oscillator's electronics (see Fig. 15). Data obtained in early 2008 from the UWA CSO (which has been kept at or below $77 \mathrm{~K}$ for over 18 months) based at NICT (Ref. 48) show an improvement in these results. The fractional frequency instability is $3 \times 10^{-15}$ at $10^{4} \mathrm{~s}$ rising to $10^{-14}$ at $10^{5} \mathrm{~s}$.

The effect of switching off various control systems on the noise properties of the CSO is illustrated by Fig. 14. It shows the importance of having the power stabilization and AM-index suppression systems for reaching the good longterm frequency stability of the oscillator.

In contrast to Ref. 38, the frequency measurements reported here were performed over the relatively long periods of time (from a few hours to a few days) in order to avoid an over optimistic estimate of the oscillator frequency stability.

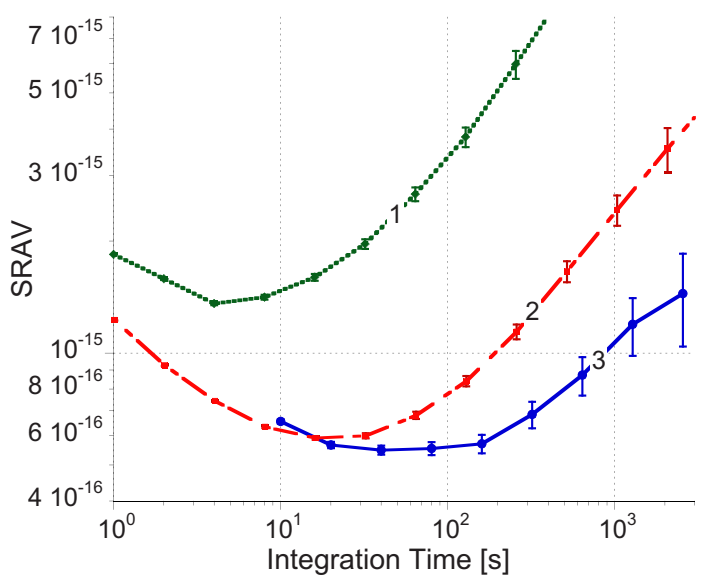

FIG. 14. (Color online) Measured Allan deviation for a single oscillator from the beat between two nominally identical cryogenic sapphire oscillators. One oscillator acted as a reference oscillator with all control systems operational. In the second oscillator power control and AM-index suppression was turned off (curve 1: gate time $1 \mathrm{~s}$ ), power control on, and AMindex off (curve 2: gate time of $1 \mathrm{~s}$ ) and both power control and AM-index suppression on (curve 3: gate time of $10 \mathrm{~s}$ ). 


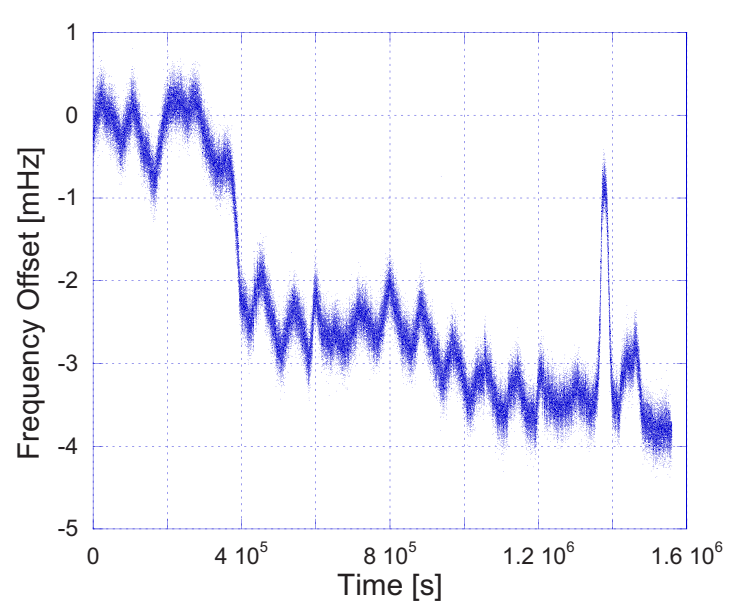

FIG. 15. (Color online) Relative beat note between a cryogenic sapphire oscillator and a synthesized signal from an environmentally isolated and temperature controlled hydrogen maser, measured over 18 days by using a countergate time of $10 \mathrm{~s}$.

The values of $\sigma_{y}$ were directly calculated from the "raw" data without any postprocessing such as a removal of the linear frequency drift typical for the earlier oscillators. ${ }^{6,7,38}$ This was due to the extremely low drift rates exhibited by our oscillators (the lowest fractional-frequency drift rate was measured to be $-2.2 \times 10^{-15} /$ day). Such performance, we believe, was due to the optimal geometry of the shielded sapphire resonator characterized by a relatively sparse spectrum of spurious modes in the vicinity of the operational one. (A low drift rate of $6 \times 10^{-14} /$ day has been reported in a microwave oscillator based on a "free space" liquid-heliumcooled sapphire-dielectric resonator. ${ }^{49}$ This is not a very practical solution, as, while eliminating the spurious cavity modes, it exposes the surface of sapphire crystal to possible contamination).

Figure 15 gives one some idea about the environmental sensitivity of the CSO. It shows the evolution of the CSO frequency relative to that of a hydrogen maser over a period of 18 days. The fuzziness of the time trace in Fig. 15 is in part due to the temperature variations in the laboratory caused by the air conditioner. The large scale periodic oscillations of the beat frequency with the magnitude of $0.6 \mathrm{mHz}$ are due to daily temperature variations in the laboratory. Two disturbances of the oscillator operation are visible in Fig. 15. The first one at time $t \approx 4 \times 10^{5} \mathrm{~s}$ corresponds to the drop of helium gas pressure in the Dewar due to the removal of the rubber stopper from the inlet pipe. The second one at $t \approx 1.38 \times 10^{6} \mathrm{~s}$ is due to some unexpected construction activity near the Dewar. A linear fit to the data between these two events gives the fractional-frequency drift rate of -7.7 $\times 10^{-15} /$ day. We have measured the performance of the UWA designed CSO based in AIST (Ref. 17) as a function of room temperature, room pressure, and helium return line pressure. In that laboratory care was taken to keep the temperature variations under $0.2{ }^{\circ} \mathrm{C}$ and the pressure variations less than $30 \mathrm{~Pa}$. These preparations resulted in a similar CSO frequency drift rate without the characteristic fuzziness or ripples seen in the UWA based CSO of Fig. 15.

\section{CONCLUSION}

We discussed the design techniques used in the construction of ultrastable microwave oscillators based on cryogenically cooled SLC resonators. These CSOs have been in continuous development over the past 18 years at the University of Western Australia and currently are the best sources of ultrastable signals with short-term fractional-frequency instability less than $6 \times 10^{-16}$ and drift rates of $-(2-5)$ $\times 10^{-15} /$ day. The unique spectral performance of the CSOs makes them suitable for a wide range of scientific applications such as the development of the laser-cooled atomic fountain clocks, very long base interferometry, and high precision tests of modern physics.

\section{ACKNOWLEDGMENTS}

The expert mechanical workshop team at UWA has provided an invaluable contribution to the CSO project. Also, we would like to acknowledge A. Luiten for his early work on the project and his continuing advice and S. Chang and A. Mann for their past contributions. Also we appreciate the support and collaborations we have experienced from NMI, Sydney; SYRTE, l'Obervatoire de Paris; CNRS; XLim, University of Limoges; CNES; FEMTO-ST; NMIJ (AIST) and NICT, Japan. This work was supported by Australian Research Council.

${ }^{1}$ V. B. Braginsky, V. P. Mitrofanov, and V. I. Panov, Systems with Small Dissipation (University of Chicago Press, Chicago, 1985).

${ }^{2}$ A. G. Mann, in Frequency Measurement and Control: Advanced Techniques and Future Trends, edited by A. N. Luiten (Springer, Berlin, 2001), pp. 37-66.

${ }^{3}$ K. S. Throne, in 300 Years of Gravitation, edited by S. W. Hawking and W. Israel (University of Chicago Press, Chicago, 1987), p. 330.

${ }^{4}$ D. G. Blair, E. N. Ivanov, M. E. Tobar, P. J. Turner, F. van Kann, and I. S. Heng, Phys. Rev. Lett. 74, 1908 (1995).

${ }^{5}$ M. E. Tobar and D. G. Blair, IEEE Trans. Microwave Theory Tech. 42, 344 (1994).

${ }^{6}$ A. J. Giles, A. G. Mann, S. K. Jones, D. G. Blair, and M. J. Buckingham, Physica B 165, 145 (1990).

${ }^{7}$ A. N. Luiten, A. G. Mann, and D. G. Blair, IEEE Trans. Instrum. Meas. 44, 132 (1995).

${ }^{8}$ M. E. Costa, D. G. Blair, M. J. Buckingham, A. J. Giles, S. K. Jones, A. N. Luiten, P. J. Turner, A. C. Young, P. Hong, and A. G. Mann, Meas. Sci. Technol. 3, 718 (1992).

${ }^{9}$ M. E. Tobar, E. N. Ivanov, C. R. Locke, P. L. Stanwix, J. G. Hartnett, A. N. Luiten, R. B. Warrington, and P. T. H. Fisk, IEEE Trans. Ultrason. Ferroelectr. Freq. Control 53, 2386 (2006).

${ }^{10}$ P. T. H. Fisk, M. J. Sellars, M. A. Lawn, C. Coles, A. G. Mann, and D. G. Blair, IEEE Trans. Instrum. Meas. 44, 113 (1995).

${ }^{11} \mathrm{See}$ http://www.crystalsystems.com/hem.html.

${ }^{12}$ G. Santarelli, Ph. Laurent, P. Lemonde, A. Clairon, A. G. Mann, S. Chang, A. N. Luiten, and C. Salomon, Phys. Rev. Lett. 82, 4619 (1999).

${ }^{13}$ P. Wolf, S. Bize, A. Clairon, A. N. Luiten, and G. Santarelli, Phys. Rev. Lett. 90, 060402 (2003).

${ }^{14}$ P. Lemonde, P. Laurent, E. Simon, G. Santarelli, A. Clairon, C. Salomon, N. Dimarcq, and P. Petit, IEEE Trans. Instrum. Meas. 48, 512 (1999).

${ }^{15}$ P. L. Stanwix, M. E. Tobar, P. Wolf, M. Susli, C. R. Locke, E. N. Ivanov, J. Winterflood, and F. van Kann, Phys. Rev. Lett. 95, 040404 (2005).

${ }^{16}$ P. L. Stanwix, M. E. Tobar, P. Wolf, C. R. Locke, and E. N. Ivanov, Phys. Rev. D 74, 081101 (R)(2006).

${ }^{17}$ K. Watabe, J. G. Hartnett, C. R. Locke, G. Santarelli, S. Yanagimachi, T. Shimazaki, T. Ikegami, and S. Ohshima, Jpn. J. Appl. Phys., Part 145 , 9234 (2006).

${ }^{18}$ K. Watabe, H. Inaba, K. Okumura, F. Hong, J. G. Hartnett, C. R. Locke, G. Santarelli, S. Yanagimachi, K. Minoshima, T. Ikegami, A. Onae, S. Ohshima, and H. Matsumoto, IEEE Trans. Instrum. Meas. 56, 632 (2007). 
${ }^{19}$ National Institute of Information and Communications Technology. See www.nict.go.jp.

${ }^{20}$ J. G. Hartnett, C. R. Locke, E. N. Ivanov, M. E. Tobar, and P. L. Stanwix, Appl. Phys. Lett. 89, 203513 (2006).

${ }^{21}$ M. E. Tobar, E. N. Ivanov, C. R. Locke, J. G. Hartnett, and D. Cros, Meas. Sci. Technol. 30, 1284 (2002).

${ }^{22}$ M. E. Tobar, G. L. Hamilton, E. N. Ivanov, and J. G. Hartnett, IEEE Trans. Ultrason. Ferroelectr. Freq. Control 50, 214 (2003).

${ }^{23}$ J. G. Hartnett, M. E. Tobar, and J. Krupka, J. Phys. D: Appl. Phys. 34, 959 (2001).

${ }^{24}$ J. G. Hartnett and M. E. Tobar, in Frequency Measurement and Control: Advanced Techniques and Future Trends edited by A. N. Luiten (Springer, Berlin, 79, 67 2001).

${ }^{25}$ G. J. Dick, D. G. Santiago, and R. T. Wang, IEEE Trans. Ultrason. Ferroelectr. Freq. Control 42, 815 (1995).

${ }^{26}$ G. J. Dick and R. T. Wang, Proceedings of the 2000 International IEEE Frequency Control Symposium, 2000 (unpublished), pp. 480-484.

${ }^{27}$ R. T. Wang, G. J. Dick, and W. A. Diener, Proceedings of the 2004 IEEE International Frequency Control Symposium, 2004 (unpublished), pp. 752-756.

${ }^{28}$ J. G. Hartnett, M. E. Tobar, A. G. Mann, E. N. Ivanov, J. Krupka, and R. Geyer, IEEE Trans. Ultrason. Ferroelectr. Freq. Control 46, 993 (1999).

${ }^{29}$ N. Boubekeur, J. G. Hartnett, M. E. Tobar, N. Bazin, Y. Kersalé, and V. Giordano, Electron. Lett. 41, 534 (2005).

${ }^{30}$ B. C. Young, F. C. Cruz, W. M. Itano, and J. C. Berquist, Phys. Rev. Lett. 82, 3799 (1999).

${ }^{31}$ M. Notcutt, L-S Ma, A. D. Ludlow, S. M. Foreman, J. Ye, and J. L. Hall, Phys. Rev. A 73, 031804 (2006).
${ }^{32}$ S. A. Webster, M. Oxborrow, and P. Gill, Phys. Rev. A 75, 011801 (2007).

${ }^{33}$ K. Numata, A. Kemery, and J. Camp, Phys. Rev. Lett. 93, 250602 (2004).

${ }^{34}$ L. Rayleigh, Philos. Mag. 20, 1001 (1910).

${ }^{35}$ M. E. Tobar and D. G. Blair, IEEE Trans. Microwave Theory Tech. 39, 1582 (1991).

${ }^{36}$ M. E. Tobar, J. Phys. D: Appl. Phys. 26, 2022 (1993).

${ }^{37}$ M. E. Tobar, A. J. Giles, S. Edwards, and J. H. Searls, IEEE Trans. Ultrason. Ferroelectr. Freq. Control 41, 391 (1994).

${ }^{38}$ S. Chang, A. Mann, and A. Luiten, Electron. Lett. 36, 480 (2000).

${ }^{39}$ M. E. Tobar and J. G. Hartnett, Phys. Rev. D 67, 062001 (2003).

${ }^{40}$ P.-Y. Bourgeois, N. Bazin, Y. Kersale, V. Giordano, M. E. Tobar, and M. Oxborrow, Appl. Phys. Lett. 87, 224104 (2005).

${ }^{41}$ R. V. Pound, Rev. Sci. Instrum. 17, 490 (1946).

${ }^{42}$ R. W. P. Drever, J. L. Hall, F. V. Kowalski, J. Hough, G. M. Ford, A. J. Munley, and H. Ward, Appl. Phys. B: Photophys. Laser Chem. 31, 97 (1983).

${ }^{43}$ S. Chang, A. G. Mann, A. N. Luiten, and D. G. Blair, Phys. Rev. Lett. 79, 2141 (1997)

${ }^{44}$ S. I. Munro, E. N. Ivanov, M. E. Tobar, P. L. Stanwix, and C. R. Locke, Meas. Sci. Technol. 15, 881 (2004)

${ }^{45}$ J. D. Anstie, J. G. Hartnett, M. E. Tobar, E. N. Ivanov, and P. L. Stanwix, IEEE Trans. Ultrason. Ferroelectr. Freq. Control 53, 284 (2006).

${ }^{46}$ D. W. Allan, Proc. IEEE 54, 221 (1966).

${ }^{47}$ J. Rutman, IEEE Trans. Instrum. Meas. 21, 85 (1972).

${ }^{48}$ M. Kumagai, H. Ito, G. Santarelli, C. R. Locke, J. G. Hartnett, and M. Hosokawa (unpublished).

${ }^{49}$ P. Y. Bourgeois, F. Lardet-Vieudrin, and Y. Kersalé, Electron. Lett. 40, 605 (2004). 